J Stroke Cerebrovasc Dis. 2014 ; 23(5): 1099-1105. doi:10.1016/j.jstrokecerebrovasdis.2013.09.019.

\title{
Correlates of Posttraumatic Stress Disorder in Stroke Survivors
}

\author{
Judith Z. Goldfinger, MD ${ }^{\mathrm{a}}$, Donald Edmondson, $\mathrm{PhD}^{\mathrm{b}}$, lan M. Kronish, MD, MPH ${ }^{\mathrm{b}}$, Kezhen \\ Fei, MS ${ }^{c}$, Revathi Balakrishnan, MD, MPH ${ }^{d}$, Stanley Tuhrim, MD $^{\mathrm{e}}$, and Carol R. Horowitz, \\ MD, MPH \\ aZena and Michael A. Wiener Cardiovascular Institute, Mount Sinai School of Medicine, New \\ York, NY, USA \\ ${ }^{\mathrm{b}}$ Center for Behavioral Cardiovascular Health, Columbia University Medical Center, New York, \\ NY, USA \\ 'Department of Health Evidence and Policy, Mount Sinai School of Medicine, New York, NY, USA \\ dDivision of Cardiology, New York University Langone Medical Center, New York, NY, USA \\ eDepartment of Neurology, Mount Sinai School of Medicine, New York, NY, USA
}

\begin{abstract}
Background-Posttraumatic stress disorder (PTSD) can occur after life threatening events, including illness, but correlates of PTSD after stroke or transient ischemic attack (TIA) have not been well described.
\end{abstract}

Methods-We measured the prevalence of stroke-induced PTSD with the PTSD ChecklistSpecific for stroke (PCL-S) in adults who had a stroke or TIA within five years. A PCL-S score $\geq$ 50 indicated likely PTSD. We tested for potential predictors of stroke-associated PTSD, including demographics, stroke history, disability, medical comorbidities, depression, and emotional support and then examined the association between post-stroke PTSD and measures of physical and mental health.

Results-Of 535 participants, 95 (18\%) had a PCL-S score $\geq 50$; the mean score was $35.4 \pm 13.7$ (range 17 to 80 out of 85 ). In logistic regression analysis, low income (OR 1.98, 95\% CI 1.013.61 ), recurrent stroke or TIA (OR 1.86, 1.10-3.16), more disability (OR 1.79, 1.43-2.23), and increased comorbidities (OR 1.90, 1.05-3.45) were independently associated with PTSD. Older age (OR 0.93, 0.90-0.95), marriage or partnership (OR 0.52, 0.28-0.98), and having emotional support (OR 0.25, 0.11-0.54) were protective against developing PTSD. Participants with likely PTSD had worse physical and mental health.

(C) 2013 National Stroke Association. Published by Elsevier Inc. All rights reserved.

Address for correspondence: Judith Z. Goldfinger, MD, Zena and Michael A. Wiener, Cardiovascular Institute, Mount Sinai School of Medicine, 1 Gustave L. Levy Place, Box 1030, New York, NY 10029; Telephone: 212-427-1540, Fax: 212-410-7196,

Judith.Goldfinger@mountsinai.org.

Disclosures: none

Publisher's Disclaimer: This is a PDF file of an unedited manuscript that has been accepted for publication. As a service to our customers we are providing this early version of the manuscript. The manuscript will undergo copyediting, typesetting, and review of the resulting proof before it is published in its final citable form. Please note that during the production process errors may be discovered which could affect the content, and all legal disclaimers that apply to the journal pertain. 
Conclusions-In this racially and ethnically diverse cohort of stroke and TIA survivors, strokeinduced PTSD was associated with younger age, recurrent strokes, greater disability and comorbidities. PTSD was associated with a substantially increased physical, mental, and quality of life burden in this already vulnerable population. Having social support was protective, suggesting a potential target for intervention.

Clinical trial registration-ClinicalTrials.gov identifier NCT01027273 http://

clinicaltrials.gov/ct2/show/study/NCT01027273?term=NCT01027273\&rank=1

\section{Keywords}

Stroke; transient ischemic attack; posttraumatic stress disorder

\section{Background}

Stroke is the third leading cause of death in the United States and the leading cause of longterm disability,(1) whether physical, cognitive, or psychological.(1-3) Psychological complications of stroke include depression in $29-33 \%$ of stroke survivors $(4,5)$ and anxiety. (6) Posttraumatic stress disorder (PTSD) is known to occur after exposure to combat or other life threatening violence, $(7,8)$ but can also develop as a result of life-threatening medical conditions as varied as HIV,(9) breast cancer,(10) acute coronary syndromes,(1113) and stroke. $(6,14-19)$

The reported prevalence of PTSD after stroke ranges from 3-37\% in studies that included up to 104 patients, who were evaluated from 3 months to 4 years after stroke. $(6,14-19)$ In these small studies, PTSD was associated with sociodemographic factors including female gender(15, 19) and limited education;(15) psychological factors including distress at the time of stroke,(19) more negative cognitive appraisals of the stroke or in general, $(15,16)$ depression and anxiety,(14); and stroke specific factors including more recent stroke or recurrent stroke.(6)

The Prevent Recurrence of All Inner-city Strokes through Education (PRAISE) trial was developed by a partnership of community leaders in Harlem, New York, and local researchers, to test the effectiveness of a peer-led, self-management course in lowering the risk for recurrent stroke among survivors of stroke and transient ischemic attack (TIA).(20) We previously reported a prevalence of PTSD of $18 \%$ among these survivors of stroke and TIA, and noted the relationship between PTSD and poor medication adherence. $(21,22) \mathrm{We}$ sought to determine correlates of PTSD in this urban population of predominantly Black and Latino, low income, stroke and TIA survivors.

\section{Methods}

Participants

Participants in the PRAISE trial were English or Spanish speaking community-dwelling adults over age 40 who self-reported a stroke or TIA within the past five years. We excluded participants whose neurologic impairments, including aphasia or cognitive deficits, would limit their ability to participate in a self-management workshop. Participants were recruited 
from senior centers, churches, and health fairs in Northern Manhattan and the Bronx, and from patient lists generated by an academic medical center, a neighborhood health center, and the Visiting Nurse Service of New York.(20) The study was approved by the Institutional Review Board of each institution and all participants provided written informed consent.

\section{Measures}

Posttraumatic stress disorder symptoms-We assessed presence of PTSD using the PTSD Checklist Specific for a stressor (PCL-S), in which participants are asked to report whether they had each symptom of PTSD as a result of their "stroke or mini-stroke."(23) The PCL-S is a validated 17-item scale that corresponds to the DSM-IV criteria for PTSD, with high internal consistency and test-retest reliability.(24-30) While studies of PTSD in civilian populations have used different cutoff points for this scale, from 30 to 50, we used the more specific cutoff of 50, which was recommended by the developers of the scale and corresponds to a sensitivity of 0.60 to 0.78 and specificity of 0.86 to 0.99 in relation to a clinical diagnosis of PTSD. $(24,25,31)$ We modified the PCL-S from a 5-point response scale ("Not at all" to "Extremely") to a 4-point response scale ("Not at all" to "Very much"), while still maintaining the score range from 17 to 85 . For analyses, we transformed PCL-S scores to reflect the standard 5-point response scale by recoding responses thus: $1=1 ; 2=$ $2.33 ; 3=3.67$; and $4=5$, then summing the responses to the 17 questions for a total PCL-S score. Scores from the modified scale should therefore still be consistent with the original scale. $(24,25)$ There were three items from the Patient Health Questionnaire (PHQ-8)(32) with substantial content overlap with items from the PCL-S: questions about loss of interest, trouble falling or staying asleep, and difficulty concentrating. We asked these questions only once, and used the responses for both the PCL-S and PHQ-8 scales. Confirmatory factor analysis results showed that these three PHQ-8 items performed as predicted, loading on first-order PTSD symptom factors with standardized factor loadings equivalent to PCL-S items.

Demographics-Age, sex, income, employment status, race and ethnicity, education, marital status, cohabitation status, insurance status, nationality, and primary language were assessed by interview.

Stroke history and related disability—Lifetime number of strokes and TIAs and the number of years since the last stroke or TIA were assessed by self-report. We assessed stroke-related disability with the modified Rankin score. $(33,34)$ This scale ranges from 0 , no disability, to 6 , dead, where 5 is severe disability requiring continuous nursing care.

Medical comorbidities-We used the Charlson comorbidity index,(35) in which a variety of medical conditions are assigned scores of 1,2,3 or 6 , resulting in a composite score. This index predicts 10-year mortality for patients with a variety of comorbid conditions, including stroke.(36)

Quality of life-Physical and mental health-related quality of life was measured with the twelve-item Medical Outcomes Short Form Health Survey (SF-12), (37, 38) which provides 
both a physical component score and a mental component score. Each is calculated using weighted scoring and standardized to a mean of 50 with a standard deviation of 10 , based on norms from the general United States population. Higher scores indicate better quality of life.

Depression-We screened for depression with the eight-item Patient Health Questionnaire (PHQ-8), a scale that assesses frequency of depression symptoms over the previous two weeks.(32, 39, 40) This modified version of the PHQ-9 excludes the question about suicidality, and had similar validity to the PHQ-9 in large epidemiologic studies. $(32,41)$ Each question is scored from 0 (not at all) to 3 (nearly every day), with total scores ranging from 0 to 24 . A score equal to or greater than ten has $88 \%$ sensitivity and $88 \%$ specificity for a diagnosis of depression.(42)

Emotional social support-Emotional social support was assessed using a single question "How many people can you count on to give you emotional support, such as talking over problems to help you with a difficult decision?" where having one or more person's support was considered presence of emotional social support.

\section{Statistical analysis}

To evaluate differences between participants with likely PTSD (PCL-S score of 50 or greater) and those without PTSD (PCL-S score 49 or less), we used the chi square test for categorical variables and studentized t-test for continuous variables. We used logistic regression to assess whether variables that predicted PTSD in other medical conditions were also significant predictors of PTSD for stroke survivors. $(6,8,14,15,43,44)$ Logistic regression included variables significant at 0.05 level from bivariate analysis, including age, sex, race/ethnicity, marital status, low income or Medicaid beneficiary, recurrent stroke or TIA, modified Rankin score, Charlson comorbidity index score, and emotional social support. All statistical analyses were performed using SAS 9.2 (SAS Institute, Carry, NC).

\section{Results}

Participants

As part of the PRAISE trial, 535 stroke and TIA survivors completed baseline assessments, including the PCL-S. The mean age of participants was 63 years, 59\% were women, $85 \%$ were Black or Latino, $30 \%$ had less than a high school education, and $55 \%$ earned less than $\$ 15,000$ annually (see Table 1). Participants had, on average, 1.7 previous strokes or TIAs, and 36\% of participants had at least two prior events (see Table 2). The mean time since the most recent event was nearly 2 years. Almost half had a modified Rankin score of 3 to 4 , consistent with moderate to moderately-severe disability. The mean number of medical conditions was 3.7. Thirty percent screened positive for depression.

\section{PTSD symptoms}

Of the 535 participants, 95 (18\%) scored 50 or higher on the PCL-S, consistent with likely PTSD. Mean PCL-S score was 35. 


\section{Unadjusted associations between PTSD status and study variables}

Compared to participants with PCL-S scores of 49 or less, stroke or TIA survivors with likely PTSD (see Table 1) were younger and more likely to be female, Black or Latino, low income, have insurance coverage through Medicaid, and report recurrent strokes or TIAs. Participants in the PTSD group were also less likely to be married or employed. There was no difference between the two groups in education level, place of birth (United States versus other), or primary language (English versus Spanish).

The PTSD group had worse stroke-related disability (Table 2): a mean modified Rankin score of 3 is consistent with an inability to perform independent activities of daily living; in contrast, the mean score of 2 in the non-PTSD group is consistent with independence in instrumental activities of daily living. While the two groups were similar in number of prior strokes and time since stroke or TIA, more participants in the PTSD group had recurrent strokes or TIAs. The PTSD group had more medical comorbidities (4.4 versus 3.5 conditions).

Presence of PTSD was associated with worse physical and mental quality of life. The PTSD group scored 32.5 on the SF-12 physical scale, nearly two full standard deviations below the population mean of 50 (see Table 2). Those without PTSD scored 39, about 1 standard deviation below the mean, despite being older (mean age 64 versus 57 years). The PTSD group also had poorer mental health-related quality of life, compared to those in the nonPTSD group: those with PTSD scored 36.9 versus 50 ( $\mathrm{p}<0.0001$ ). Participants in the PTSD were more likely to be depressed: $78 \%$ of the PTSD group was depressed, versus $19 \%$ of the group without PTSD ( $<<0.0001)$.

\section{Logistic regression predicting positive PTSD screen}

Results of the logistic regression analysis (see Table 3) showed that younger age (odds ratio 0.93 for age, $95 \%$ confidence interval $0.90-0.95$ ), recurrent stroke or TIA (OR 1.86, 95\% CI 1.20-3.16), greater stroke-related disability with Rankin score of 3-4 versus $0-2$ (OR 1.79, 95\% CI 1.43-2.23), three or more medical comorbidities versus fewer than two (OR 1.90, 95\% CI 1.05-3.45), unmarried status (OR 0.52, 95\% CI 0.28-0.98 for married or unmarried partnership), and lack of emotional social support (OR $0.25,95 \%$ CI $0.11-0.54$ for presence of emotional social support) were independently associated with greater odds of screening positive for PTSD. Sex and race were not significant predictors of PTSD.

\section{Discussion}

In this large, ethnically diverse cohort of stroke and TIA survivors, younger survivors, with recurrent stroke or TIA, greater stroke-related disability, and greater number of medical conditions were more likely to develop PTSD. Markers of social support, including reporting at least one person who provides emotional support, or being married or in an unmarried partnership, were protective against development of PTSD. While race and sex were significant predictors in unadjusted analyses, these covariates were not significant after adjustment for other demographic and clinical variables. PTSD was also associated with 
worse physical and mental health and worse health-related quality of life. Interestingly, there was no relationship between PTSD and time since stroke.

Some of the correlates of PTSD identified in this study were noted in previous studies: Merriman and colleagues also identified recurrent strokes as a predictor of PTSD.(6) Similar to our finding, social support was protective against the development of PTSD for both combat veterans and survivors of the September 11 attacks. $(8,43,44)$ The relationship between PTSD and worse mental health was suggested in previous small studies of stroke survivors, where PTSD was associated with depression and anxiety, $(14,17)$ as well as negative affect or negative cognitive appraisal in general or of the stroke in particular.(6, 15, $16,18,19)$ To our knowledge, this is the largest study to date to examine the correlates of PTSD in a post-stroke population.

It is important to identify and treat stroke and TIA survivors who are at risk for PTSD. In stable cardiovascular disease, PTSD is associated with worse health outcomes, including worse physical and cardiovascular health status.(45) In large cohort studies, PTSD symptoms were associated with an increased risk for both incident and recurrent cardiovascular disease. $(12,46,47)$ In non-stroke populations, PTSD is associated with increased blood pressure, systemic inflammation, and other physiological markers of stroke risk.(48, 49) PTSD also impacts health behaviors, including worse medication adherence in stroke survivors, $(21,22)$ which may increase risk for recurrent stroke or other cardiovascular events.

Given the high prevalence of PTSD in our study and the concern about PTSD impacting health outcomes, physicians who care for stroke patients should screen for PTSD, especially in those patients who may be at higher risk as suggested by this study: younger patients and those with more strokes, greater disability, and more medical conditions. We also found that social support, whether from a friend, spouse, or partner, was protective against developing PTSD, suggesting a role for interventions that decrease social isolation among stroke survivors.

Limitations of this study include the use of a screening tool instead of a structured clinical interview to measure PTSD. We chose a highly specific cutoff that corresponds to a specificity of 0.86 to 0.99 when compared with a clinical interview. $(24,25,31)$ Further, we used a modified form of the PCL-S by allowing items with substantial content overlap from the PHQ to stand in for PCL-S items. However, internal consistency, reliability, and confirmatory factor analysis suggested that our decision did not impact the measurement of PTSD symptoms. While there are more extensive scales, we chose the PCL-S for its excellent diagnostic utility, brevity, ease of use, ubiquity in the medical literature, and use of specific DSM-IV criteria for PTSD.(24-30) We also used self-report to assess sociodemographics and markers of physical and mental health. Another possible limitation is whether these findings are generalizable to the broader community of stroke survivors, as our study population was predominantly Black or Latino and of low socioeconomic status, and had agreed to participate in a randomized trial to test an intervention intended to improve adherence. 
Our study helps create a portrait of PTSD after stroke in this urban, predominantly minority population: young, isolated, disabled, sick, with recurrent strokes or TIAs. The most vulnerable survivors, namely those with poorer physical and mental health, are more likely to be affected, and the direction of this relationship requires further exploration. Further research is needed to explore the effects of PTSD on health outcomes in stroke survivors, and to investigate the effects of interventions to prevent or treat PTSD in this population.

\section{Acknowledgments}

The study authors would like to thank Zakia Griffin, Emily Purchia, Latisha Ricketts, Gabriela Sanchez-Bravo, and Yohana Taveras for their role in data collection; Rennie Negron who served as Project Manager; and Guedy Arniella for her assistance with the PRAISE trial.

Funding sources: National Institute on Minority Health and Health Disparities (P60MD000270) and the National Center for Research Resources (UL1RR029887) of the National Institutes of Health. The funders were not involved in study design; in the collection, analysis and interpretation of data; in the writing of the report; or in the decision to submit the article for publication.

Grant support: Dr. Horowitz, Dr. Goldfinger, and Ms. Fei received support from the National Institute of Minority Health and Health Disparities (P60MD00270), and Dr. Horowitz received funding from the National Center for Research Resources (UL1RR029887). Dr. Edmondson is supported by grants KM1 CA-156709 and HL-088117 from the National Institutes of Health. Dr. Kronish received support from the National Heart, Lung and Blood Institute (K23 HL098359).

\section{References}

1. Roger VL, Go AS, Lloyd-Jones DM, et al. Heart disease and stroke statistics--2012 update: A report from the American heart association. Circulation. 2012; 125:e2-e220. [PubMed: 22179539]

2. Furie KL, Kasner SE, Adams RJ, et al. Guidelines for the prevention of stroke in patients with stroke or transient ischemic attack: A guideline for healthcare professionals from the American heart association/American stroke association. Stroke. 2011; 42:227-76. [PubMed: 20966421]

3. Haley WE, Roth DL, Kissela B, et al. Quality of life after stroke: A prospective longitudinal study. Qual Life Res. 2011; 20:799-806. [PubMed: 21136298]

4. Hackett ML, Yapa C, Parag V, et al. Frequency of depression after stroke: A systematic review of observational studies. Stroke. 2005; 36:1330-40. [PubMed: 15879342]

5. Ayerbe L, Ayis S, Wolfe CD, et al. Natural history, predictors and outcomes of depression after stroke: Systematic review and meta-analysis. Br J Psychiatry. 2013; 202:14-21. cited 2/18/2013 12:50:04 PM. [PubMed: 23284148]

6. Merriman C, Norman P, Barton J. Psychological correlates of PTSD symptoms following stroke. Psychol Health Med. 2007; 12:592-602. [PubMed: 17828679]

7. Health status of Vietnam veterans. I. psychosocial characteristics. the centers for disease control Vietnam experience study. JAMA. 1988; 259:2701-7. [PubMed: 2833630]

8. Polusny MA, Erbes CR, Murdoch M, et al. Prospective risk factors for new-onset post-traumatic stress disorder in national guard soldiers deployed to Iraq. Psychol Med. 2011; 41:687-98. [PubMed: 21144108]

9. Sherr L, Nagra N, Kulubya G, et al. HIV infection associated post-traumatic stress disorder and post-traumatic growth--a systematic review. Psychol Health Med. 2011; 16:612-29. [PubMed: 21793667]

10. O'Connor M, Christensen S, Jensen AB, et al. How traumatic is breast cancer? post-traumatic stress symptoms (PTSS) and risk factors for severe PTSS at 3 and 15 months after surgery in a nationwide cohort of Danish women treated for primary breast cancer. Br J Cancer. 2011; 104:419-26. [PubMed: 21224851]

11. Bennett $\mathrm{P}$, Brooke $\mathrm{S}$. Intrusive memories, post-traumatic stress disorder and myocardial infarction. Br J Clin Psychol. 1999; 38:411-6. [PubMed: 10590829] 
12. Edmondson D, Rieckmann N, Shaffer JA, et al. Posttraumatic stress due to an acute coronary syndrome increases risk of 42-month major adverse cardiac events and all-cause mortality. $\mathbf{J}$ Psychiatr Res. 2011; 45:1621-6. [PubMed: 21807378]

13. Edmondson D, Richardson S, Falzon L, et al. Posttraumatic stress disorder induced by acute coronary syndrome: A meta-analytic review of prevalence and associated clinical outcomes. PLoS ONE. 2012; 7:e38915. [PubMed: 22745687]

14. Sembi S, Tarrier N, O’Neill P, et al. Does post-traumatic stress disorder occur after stroke: A preliminary study. Int J Geriatr Psychiatry. 1998; 13:315-22. [PubMed: 9658264]

15. Bruggimann L, Annoni JM, Staub F, et al. Chronic posttraumatic stress symptoms after nonsevere stroke. Neurology. 2006; 66:513-6. [PubMed: 16505303]

16. Field EL, Norman P, Barton J. Cross-sectional and prospective associations between cognitive appraisals and posttraumatic stress disorder symptoms following stroke. Behav Res Ther. 2008; 46:62-70. [PubMed: 18005937]

17. Sagen U, Vik TG, Moum T, et al. Screening for anxiety and depression after stroke: Comparison of the hospital anxiety and depression scale and the Montgomery and Asberg depression rating scale. J Psychosom Res. 2009; 67:325-32. [PubMed: 19773025]

18. Wang X, Chung MC, Hyland ME, et al. Posttraumatic stress disorder and psychiatric co-morbidity following stroke: The role of alexithymia. Psychiatry Res. 2011; 188:51-7. [PubMed: 21036403]

19. Letamendia C, Leblanc NJ, Pariente J, et al. Peritraumatic distress predicts acute posttraumatic stress disorder symptoms after a first stroke. Gen Hosp Psychiatry. 2012; 34:e11-3. [PubMed: 22542052]

20. Goldfinger JZ, Kronish IM, Fei K, et al. Peer education for secondary stroke prevention in innercity minorities: Design and methods of the prevent recurrence of all inner-city strokes through education randomized controlled trial. Contemp Clin Trials. 2012

21. Kronish IM, Edmondson D, Goldfinger JZ, et al. Posttraumatic stress disorder and adherence to medications in survivors of strokes and transient ischemic attacks. Stroke. 2012:43. [PubMed: 23250997]

22. Edmondson D, Horowitz CR, Goldfinger JZ, et al. Concerns about medications mediate the association of posttraumatic stress disorder with adherence to medication in stroke survivors. $\mathrm{Br} \mathrm{J}$ Health Psychol. 2013 cited 2/18/2013 12:45:49 PM.

23. Weathers, F.; Litz, B.; Herman, D.; Huska, J.; Keane, T. The PTSD checklist: Reliability, validity, $\&$ diagnostic utility. San Antonio, TX. Annual Meeting of the International Society of Traumatic Stress Studies; 1993.

24. Blanchard EB, Jones-Alexander J, Buckley TC, et al. Psychometric properties of the PTSD checklist (PCL). Behav Res Ther. 1996; 34:669-73. [PubMed: 8870294]

25. Ruggiero KJ, Del Ben K, Scotti JR, et al. Psychometric properties of the PTSD checklist-civilian version. J Trauma Stress. 2003; 16:495-502. [PubMed: 14584634]

26. Elhai JD, Gray MJ, Kashdan TB, et al. Which instruments are most commonly used to assess traumatic event exposure and posttraumatic effects? : A survey of traumatic stress professionals. J Trauma Stress. 2005; 18:541-5. [PubMed: 16281252]

27. Adkins JW, Weathers FW, McDevitt-Murphy M, et al. Psychometric properties of seven selfreport measures of posttraumatic stress disorder in college students with mixed civilian trauma exposure. J Anxiety Disord. 2008; 22:1393-402. [PubMed: 18436427]

28. Miles JN, Marshall GN, Schell TL. Spanish and English versions of the PTSD checklist-civilian version (PCL-C): Testing for differential item functioning. J Trauma Stress. 2008; 21:369-76. [PubMed: 18720394]

29. Kelley LP, Weathers FW, McDevitt-Murphy ME, et al. A comparison of PTSD symptom patterns in three types of civilian trauma. J Trauma Stress. 2009; 22:227-35. [PubMed: 19444884]

30. Conybeare D, Behar E, Solomon A, et al. The PTSD checklist-civilian version: Reliability, validity, and factor structure in a nonclinical sample. J Clin Psychol. 2012; 68:699-713. [PubMed: 22517497]

31. Andrykowski MA, Cordova MJ, Studts JL, et al. Posttraumatic stress disorder after treatment for breast cancer: Prevalence of diagnosis and use of the PTSD checklist-civilian version (PCL-C) as a screening instrument. J Consult Clin Psychol. 1998; 66:586-90. [PubMed: 9642900] 
32. Kroenke K, Strine TW, Spitzer RL, et al. The PHQ-8 as a measure of current depression in the general population. J Affect Disord. 2009; 114:163-73. [PubMed: 18752852]

33. van Swieten JC, Koudstaal PJ, Visser MC, et al. Interobserver agreement for the assessment of handicap in stroke patients. Stroke. 1988; 19:604-7. [PubMed: 3363593]

34. Wilson JT, Hareendran A, Grant M, et al. Improving the assessment of outcomes in stroke: Use of a structured interview to assign grades on the modified Rankin scale. Stroke. 2002; 33:2243-6. [PubMed: 12215594]

35. Charlson ME, Pompei P, Ales KL, et al. A new method of classifying prognostic comorbidity in longitudinal studies: Development and validation. J Chronic Dis. 1987; 40:373-83. [PubMed: 3558716]

36. Goldstein LB, Samsa GP, Matchar DB, et al. Charlson index comorbidity adjustment for ischemic stroke outcome studies. Stroke. 2004; 35:1941-5. [PubMed: 15232123]

37. Ware J Jr, Kosinski M, Keller SD. A 12-item short-form health survey: Construction of scales and preliminary tests of reliability and validity. Med Care. 1996; 34:220-33. [PubMed: 8628042]

38. Okonkwo OC, Roth DL, Pulley L, et al. Confirmatory factor analysis of the validity of the SF-12 for persons with and without a history of stroke. Qual Life Res. 2010; 19:1323-31. [PubMed: 20567914]

39. Williams LS, Brizendine EJ, Plue L, et al. Performance of the PHQ-9 as a screening tool for depression after stroke. Stroke. 2005; 36:635-8. [PubMed: 15677576]

40. American Psychiatric Association. Diagnostic and statistical manual of mental disorders. 4. Washington, DC: American Psychiatric Association; 2000. Text Revision

41. Corson K, Gerrity MS, Dobscha SK. Screening for depression and suicidality in a VA primary care setting: 2 items are better than 1 item. Am J Manag Care. 2004; 10:839-45. [PubMed: 15609737]

42. Kroenke K, Spitzer RL, Williams JB. The PHQ-9: Validity of a brief depression severity measure. J Gen Intern Med. 2001; 16:606-13. [PubMed: 11556941]

43. DiGrande L, Perrin MA, Thorpe LE, et al. Posttraumatic stress symptoms, PTSD, and risk factors among lower Manhattan residents 2-3 years after the September 11, 2001 terrorist attacks. J Trauma Stress. 2008; 21:264-73. [PubMed: 18553414]

44. DiGrande L, Neria Y, Brackbill RM, et al. Long-term posttraumatic stress symptoms among 3,271 civilian survivors of the September 11, 2001, terrorist attacks on the world trade center. Am J Epidemiol. 2011; 173:271-81. [PubMed: 21190987]

45. Cohen BE, Marmar CR, Neylan TC, et al. Posttraumatic stress disorder and health-related quality of life in patients with coronary heart disease: Findings from the heart and soul study. Arch Gen Psychiatry. 2009; 66:1214-20. [PubMed: 19884609]

46. Kubzansky LD, Koenen KC. Is posttraumatic stress disorder related to development of heart disease? an update. Cleve Clin J Med. 2009; 76 (Suppl 2):S60-5. [PubMed: 19376986]

47. Coughlin SS. Post-traumatic stress disorder and cardiovascular disease. Open Cardiovasc Med J. 2011; 5:164-70. [PubMed: 21792377]

48. Yehuda R. Post-traumatic stress disorder. N Engl J Med. 2002; 346:108-14. [PubMed: 11784878]

49. Bedi US, Arora R. Cardiovascular manifestations of posttraumatic stress disorder. J Natl Med Assoc. 2007; 99:642-9. [PubMed: 17595933] 
Table 1

Association between PTSD and Socio-demographic Characteristics

\begin{tabular}{|c|c|c|c|c|}
\hline & Total $(\mathrm{N}=535)$ & PTSD (PCL- $S \unrhd 0)(\mathrm{N}=95)$ & No PTSD (PCL- $\mathrm{S}<50)(\mathrm{N}=440)$ & p-value \\
\hline Age, mean in years $\pm \mathrm{SD}$ & $63.0 \pm 11.3$ & $57.2 \pm 8.5$ & $64.2 \pm 11.5$ & $<0.001$ \\
\hline Female gender & $317(59.3 \%)$ & $65(68.4 \%)$ & $252(57.3 \%)$ & 0.04 \\
\hline Black or Latino* & $422(84.7 \%)$ & $88(97.8 \%)$ & $334(81.9 \%)$ & 0.0001 \\
\hline Less than high school education & $157(29.6 \%)$ & $25(26.9 \%)$ & $132(30.1 \%)$ & 0.53 \\
\hline Married or unmarried partners & $192(36.0 \%)$ & $21(22.1 \%)$ & $171(39.0 \%)$ & 0.002 \\
\hline Live alone & $185(34.6 \%)$ & $40(42.1 \%)$ & $145(33.0 \%)$ & 0.089 \\
\hline Employment & & & & $<.0001$ \\
\hline Working full- or part-time & $76(14.3 \%)$ & $7(7.4 \%)$ & $69(15.8 \%)$ & \\
\hline Unable to work & $205(38.5 \%)$ & $64(67.4 \%)$ & $141(32.2 \%)$ & \\
\hline Retired, in school, or other & $252(47.3 \%)$ & $24(25.3 \%)$ & $228(52.1 \%)$ & \\
\hline Yearly income $<\$ 15,000$ & $274(55.4 \%)$ & $68(77.3 \%)$ & $206(50.6 \%)$ & $<0.0001$ \\
\hline Insurance & & & & 0.0002 \\
\hline Commercial & $64(12.0 \%)$ & $11(11.7 \%)$ & $53(12.1 \%)$ & \\
\hline Medicare & $279(52.4 \%)$ & $33(35.1 \%)$ & $246(56.2 \%)$ & \\
\hline Medicaid or uninsured & $171(32.1 \%)$ & $48(51.1 \%)$ & $123(28.1 \%)$ & \\
\hline Born in the United States & $325(60.9 \%)$ & $56(59.0 \%)$ & $269(61.3 \%)$ & 0.67 \\
\hline Primary language & & & & 0.88 \\
\hline English & $408(76.3 \%)$ & $73(76.8 \%)$ & $335(76.1 \%)$ & \\
\hline Spanish & $127(23.7 \%)$ & $22(23.2 \%)$ & $105(23.9 \%)$ & \\
\hline
\end{tabular}


Table 2

Association between PTSD status and physical and mental health

\begin{tabular}{lcccc}
\hline & Total (N=535) & PTSD (N=95) & No PTSD (N=440) & p-value \\
\hline PTSD score (PCL-S) & $35.4 \pm 13.7$ & $59.1 \pm 7.6$ & $30.3 \pm 8.3$ & \\
Physical Health & & & & \\
Number of strokes or TIAs & $1.7 \pm 1.2$ & $1.8 \pm 1.3$ & $1.6 \pm 1.2$ & 0.13 \\
$\quad$ Had more than one stroke or TIA, n (\%) & $191(35.7 \%)$ & $44(46.3 \%)$ & $147(33.4 \%)$ & 0.02 \\
Years since most recent stroke or TIA & $1.9 \pm 1.4$ & $1.7 \pm 1.4$ & $1.9 \pm 1.5$ & 0.17 \\
Modified Rankin score & $2.1 \pm 1.4$ & $2.9 \pm 1.1$ & $1.9 \pm 1.4$ & $<0.001$ \\
Charlson comorbidity index & $3.7 \pm 2.2$ & $4.4 \pm 2.3$ & $3.5 \pm 2.1$ & 0.001 \\
SF-12 Physical Component score & $37.9 \pm 11.6$ & $32.5 \pm 10.1$ & $39.0 \pm 11.5$ & $<0.0001$ \\
Mental Health & & & & \\
Depression score (PHQ-8) & $7.3 \pm 5.5$ & $13.9 \pm 4.8$ & $5.8 \pm 4.6$ & $<0.0001$ \\
$\quad$ Depressed (PHQ-8 $\geq 10), \mathrm{n}(\%)$ & $159(29.7 \%)$ & $74(77.9 \%)$ & $85(19.3 \%)$ & $<0.0001$ \\
SF-12 Mental Component score & $47.7 \pm 12.5$ & $36.9 \pm 10.5$ & $50.0 \pm 11.7$ & $<0.0001$ \\
Emotional social support & $488(91.2 \%)$ & $79(83.2 \%)$ & $409(93.0 \%)$ & 0.002 \\
\hline
\end{tabular}

Note: Summary statistics are given as mean \pm 1 standard deviation unless otherwise noted. 
Table 3

Logistic regression predicting positive PTSD screen from demographic, clinical, and social support variables

\begin{tabular}{lcc}
\hline Covariate & Odds Ratio & 95\% \\
\hline Age & 0.93 & $0.90-0.95$ \\
Male sex & 0.91 & $0.52-1.59$ \\
White race & 0.37 & $0.08-1.74$ \\
Married or unmarried partners & 0.52 & $0.28-0.98$ \\
Low income or Medicaid beneficiary & 1.98 & $1.08-3.61$ \\
Had more than one stroke or TIA & 1.86 & $1.10-3.16$ \\
Rankin score 3-4 & 1.79 & $1.43-2.23$ \\
Charlson medical comorbidity score $\geq 3$ & 1.90 & $1.05-3.45$ \\
Presence of emotional social support & 0.25 & $0.11-0.54$ \\
\hline
\end{tabular}

Model c-statistic: $0.84 ;$ Wald $(9)=71.16, \mathrm{p}<0.0001$. 